\title{
Bright Mid-Wave Infrared Resonant-Cavity Light-Emitting Diodes Based on Black Phosphorus
}

Niharika Gupta ${ }^{1,2}$, Hyungjin Kim ${ }^{1,2}$, Nima Sefidmooye Azar $^{3,4}$, Shiekh Zia Uddin ${ }^{1,2}$, Der-Hsien Lien $^{1,2}$, Kenneth B. Crozier ${ }^{3,4,5}$, Ali Javey ${ }^{1,2 *}$

${ }^{1}$ Electrical Engineering \& Computer Sciences, University of California, Berkeley, CA 94720, USA

${ }^{2}$ Materials Sciences Division, Lawrence Berkeley National Laboratory, Berkeley, CA 94720, USA

${ }^{3}$ Department of Electrical and Electronic Engineering, University of Melbourne, Victoria 3010, Australia

${ }^{4}$ Australian Research Council (ARC) Centre of Excellence for Transformative Meta-Optical Systems, University of Melbourne, Victoria 3010, Australia

${ }^{5}$ School of Physics, University of Melbourne, Victoria 3010, Australia

*Correspondence should be addressed to A.J. (ajavey@berkeley.edu) 


\section{S1: Fabrication of bP-LED}

Firstly, the optical cavity was fabricated by firstly depositing Ti/Au/Ti $(5 \mathrm{~nm} / 120 \mathrm{~nm} / 5 \mathrm{~nm})$ using ebeam evaporation followed by sputtering $360 \mathrm{~nm} \mathrm{Al}{ }_{2} \mathrm{O}_{3}$. $\mathrm{MoS}_{2}$ was mechanically exfoliation on $50 \mathrm{~nm} \mathrm{SiO} / 2 \mathrm{Si}$ substrate onto which $10 \mathrm{~nm}$ ITO was sputtered. bP (Smart Elements) due to its air sensitivity was exfoliated in a glove box. The thicknesses of the flakes were measured using AFM (Atomic Force Microscopy, Bruker Dimension Icon with ScanAsyst) and the stack was prepared using dry-transfer technique. Utilizing poly (methyl methacrylate) or PMMA as carrier, the $\mathrm{MoS}_{2} / \mathrm{ITO}$ stack was lifted and transferred to the bP, after which the whole stack was placed onto the cavity to create the final structure. Next, the contacts were patterned using the high-resolution write-head in the uPG-101 Laser Lithography System, followed by thermal evaporation of $40 \mathrm{~nm}$ $\mathrm{Ni}$ contacts and overnight liftoff in anhydrous acetone (in glovebox) to conclude the fabrication process. 


\section{S2: Raman Spectroscopy}

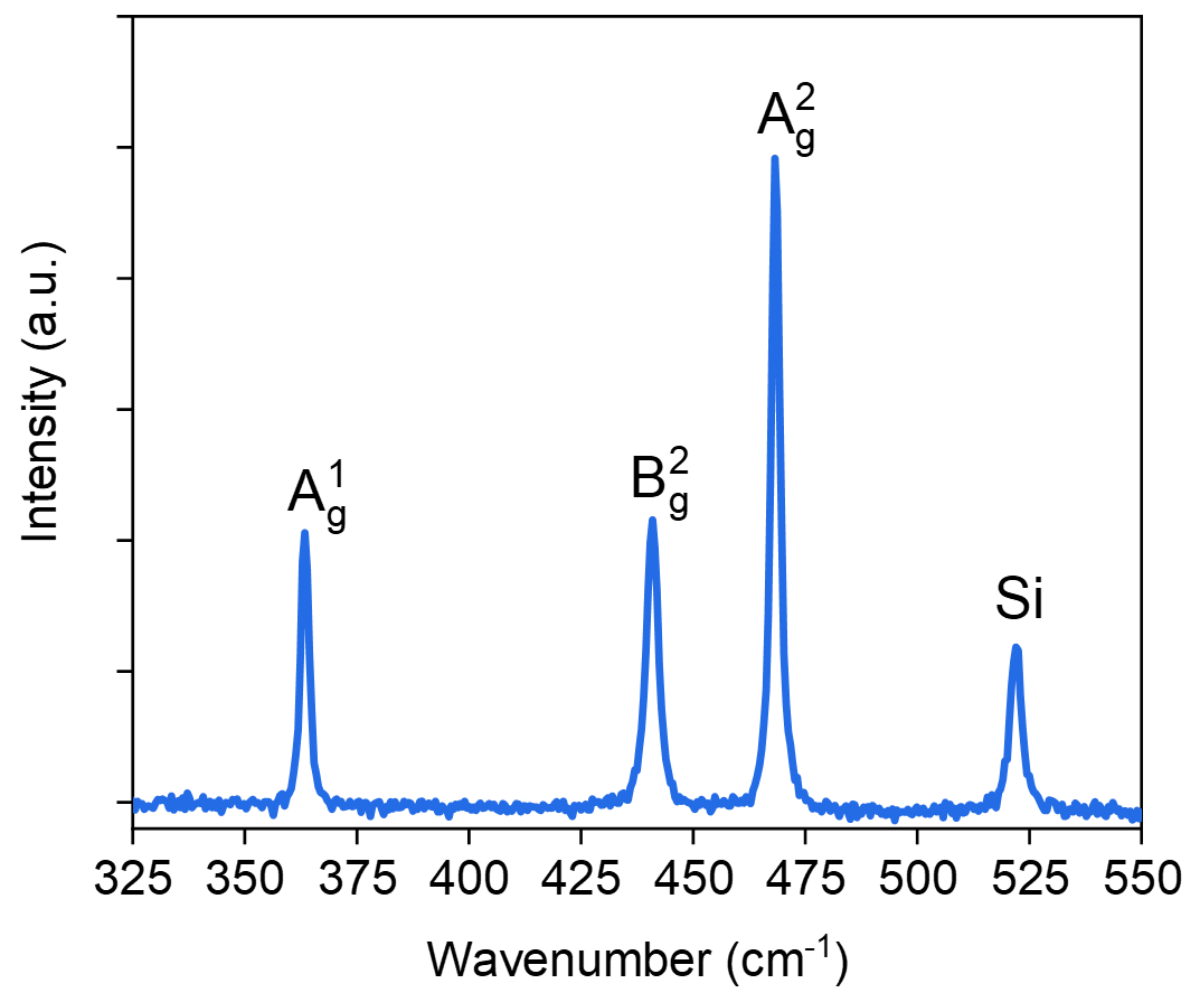

Fig. S1. Raman spectrum of exfoliated bP flake. 


\section{S3: Device Electrical and Optical Measurements}

Electrical measurements ( $I-V$ characteristics) were performed using Agilent $4155 \mathrm{C}$ Semiconductor Parameter Analyzer and the junction area was analyzed using ImageJ software. Sheet resistance of ITO was measured using Guardian SRM-232. For the estimation of input carrier and power density, and output power density, it was assumed that distribution is uniform across the junction area. For the optical measurements, devices were wire-bonded in a 24-pin chip carrier and placed in a Janis cryostat evacuated to a base pressure $<10^{-4}$ Torr. One of the major challenges in infrared emitter measurements is the separation of the electroluminescence of the diode from the thermal background, therefore, a double modulation scheme was used to solve this problem. ${ }^{6}$ The first modulation was executed using function generator (Agilent 81150) inputting voltage at a frequency of $5 \mathrm{kHz}$ and $50 \%$ duty cycle to a voltage controlled current source (CS589 Stanford Research Systems) injecting current into the device inside the cryostat. The modulated light output from the device passed through a $\mathrm{CaF}_{2}$ window in the cryostat and then a $15 \times$ Schwarzschild objective to the external source port of FTIR (iS50, Thermo Fisher) where the emitted light was modulated for a second time. The beam splitter and detector used for these measurements were $\mathrm{CaF}_{2}$ and liquid- $\mathrm{N}_{2}$ cooled $\mathrm{HgCdTe}$ (MCT) detector respectively and the FTIR optical velocity was set at $0.0657 \mathrm{~cm} \mathrm{~s}^{-1}$. The signal from the FTIR was de-modulated using a lock-in amplifier (SR865) which locked-in the signal at $5 \mathrm{kHz}$ (the frequency of the injected current) and outputted the separated signal from the background to the oscilloscope. Thereafter, the data was acquired and processed in a custom-built LabView program in which Fast Fourier Transform (FFT) was performed to convert the signal from interferogram in the time-domain to the frequency-domain to collect the bP-LED emission spectrum. The cryostat was mounted on translation stages and x-, $y$ - and z-controllers were adjusted to maximize the signal from the device. The setup schematic has been shown in Fig. S2. For the drift measurement, the device was modulated at a frequency of 
$1 \mathrm{kHz}$, and the de-modulated signal from the lock-in amplifier was sampled at frequency of $1 \mathrm{~s}$ and recorded in a different LabView program.

1. Fuchs, F., Lusson, A., Wagner, J., \& Koidl, P. (1989, December). Double modulation techniques in Fourier transform infrared photoluminescence. In 7th Intl Conf on Fourier Transform Spectroscopy (Vol. 1145, pp. 323-326). International Society for Optics and Photonics.

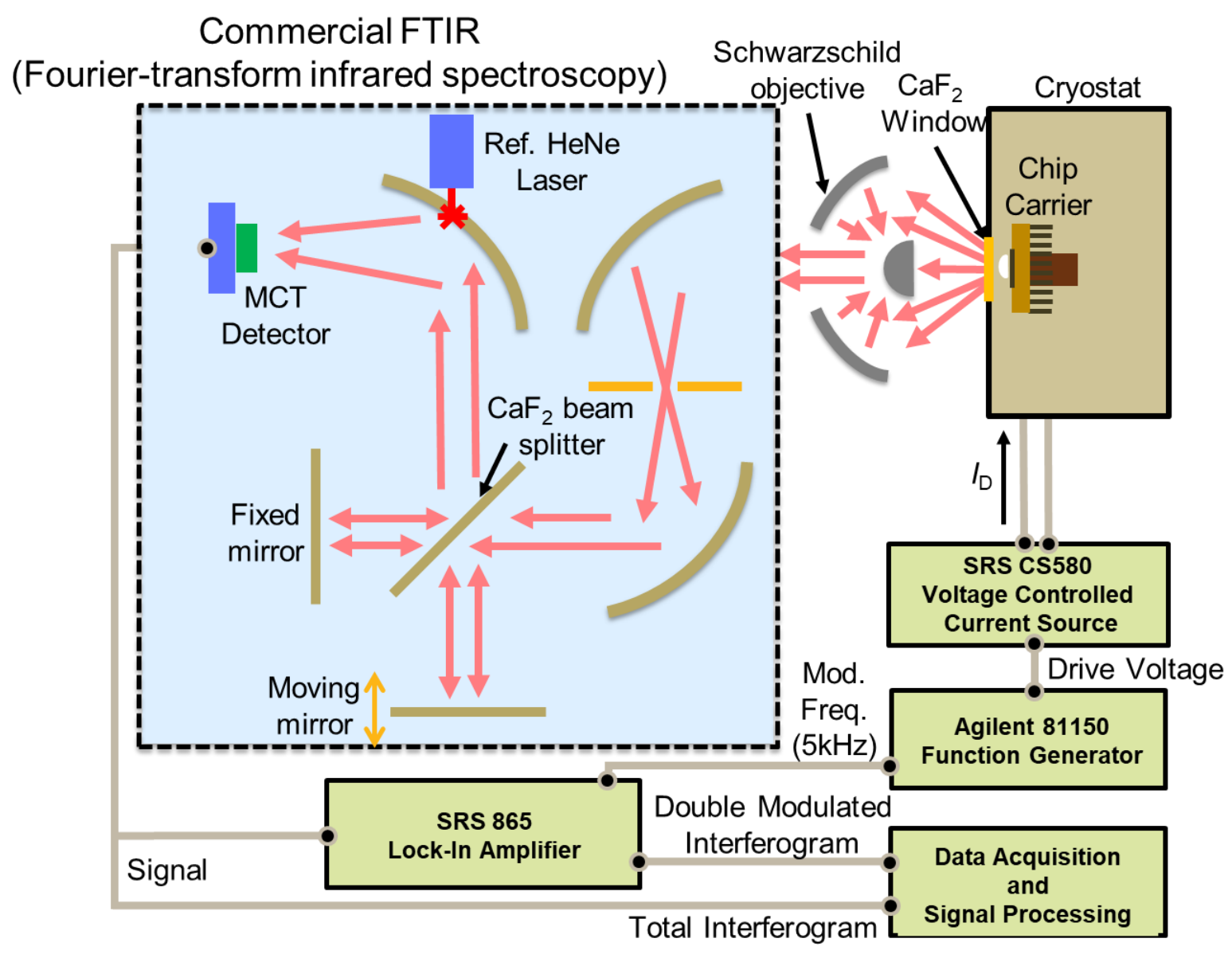

Fig. S2. Schematic of the measurement setup. 


\section{S4: Polarized Electroluminescence}

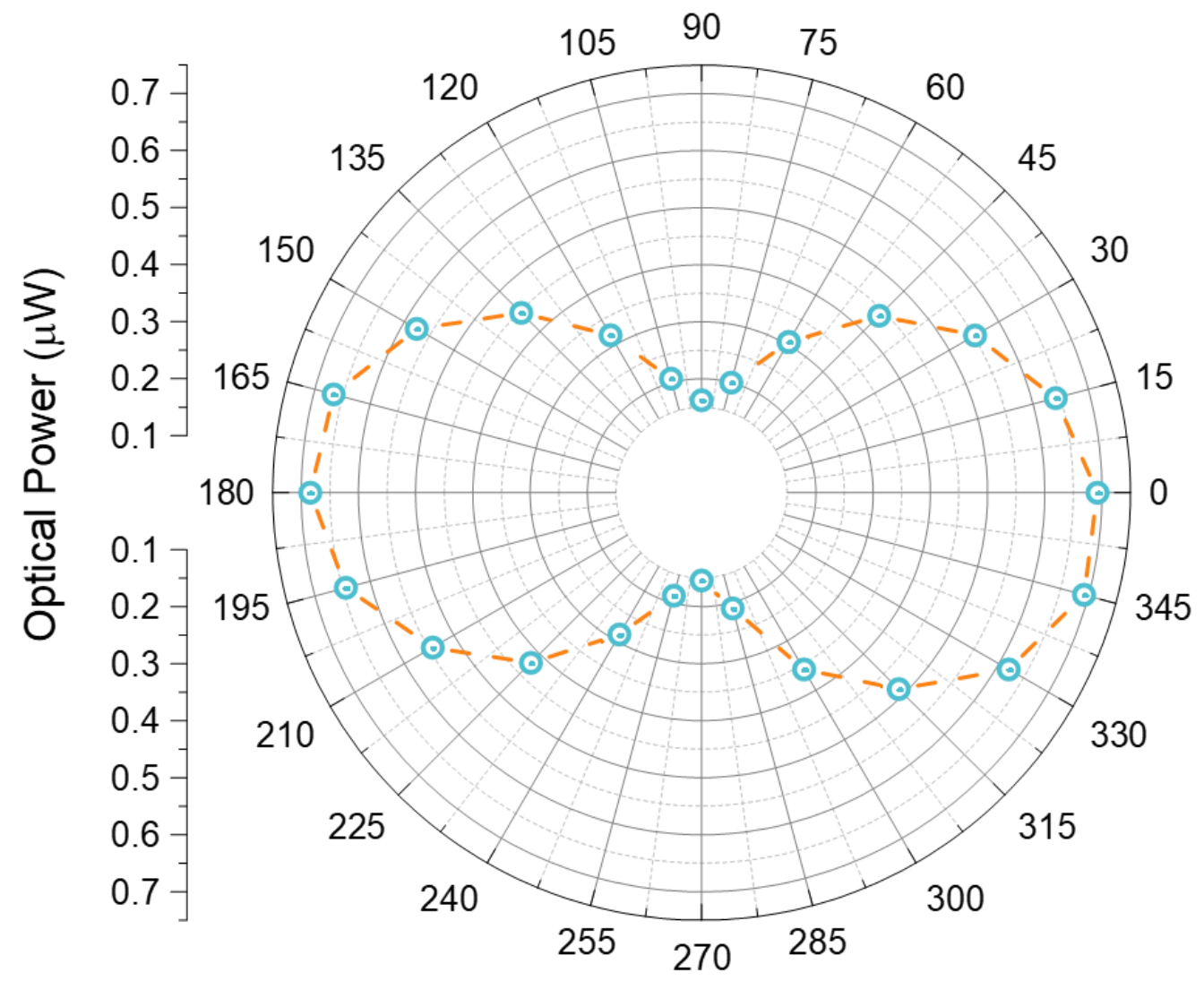

Fig. S3. Polarized Optical Power from bP-LED as a function of polarization angle. 


\section{S5: Optical Simulations Setup}

The simulations to extract the outcoupling efficiency and Purcell Factor were modelled in Lumerical FDTD Solutions. The refractive index data of $\mathrm{Au}$ and $\mathrm{Al}_{2} \mathrm{O}_{3}$ was used from the material data base in the software, and refractive index of $\mathrm{MoS}_{2}$ chosen as 4.227. The structure was setup to mimic the device structure with the thicknesses used for the experiments. The light source was chosen as a dipole placed in bP, whose peak wavelength changed with the bP thickness (thickness changes the optical bandgap for bP). To eliminate the errors caused by the dipole source in the infrared absorbing media, the imaginary refractive index of bP was set to 0 and the real part of the refractive index used was 3.997 (xx), 2.891 (yy) and 3.575 (zz), owing to the anisotropic properties of the material. Mesh size was chosen such that each layer had at least 10 elements and the boundary conditions were used as perfectly matched layers (PMLs). Purcell factor was computed using the transmission through the source monitor box tightly enclosing the dipole source and the outcoupling efficiency was determined by the ratio of the transmission of far-field monitor to that through the source monitor. 
S6: Effect of bP Thickness on Optical Power

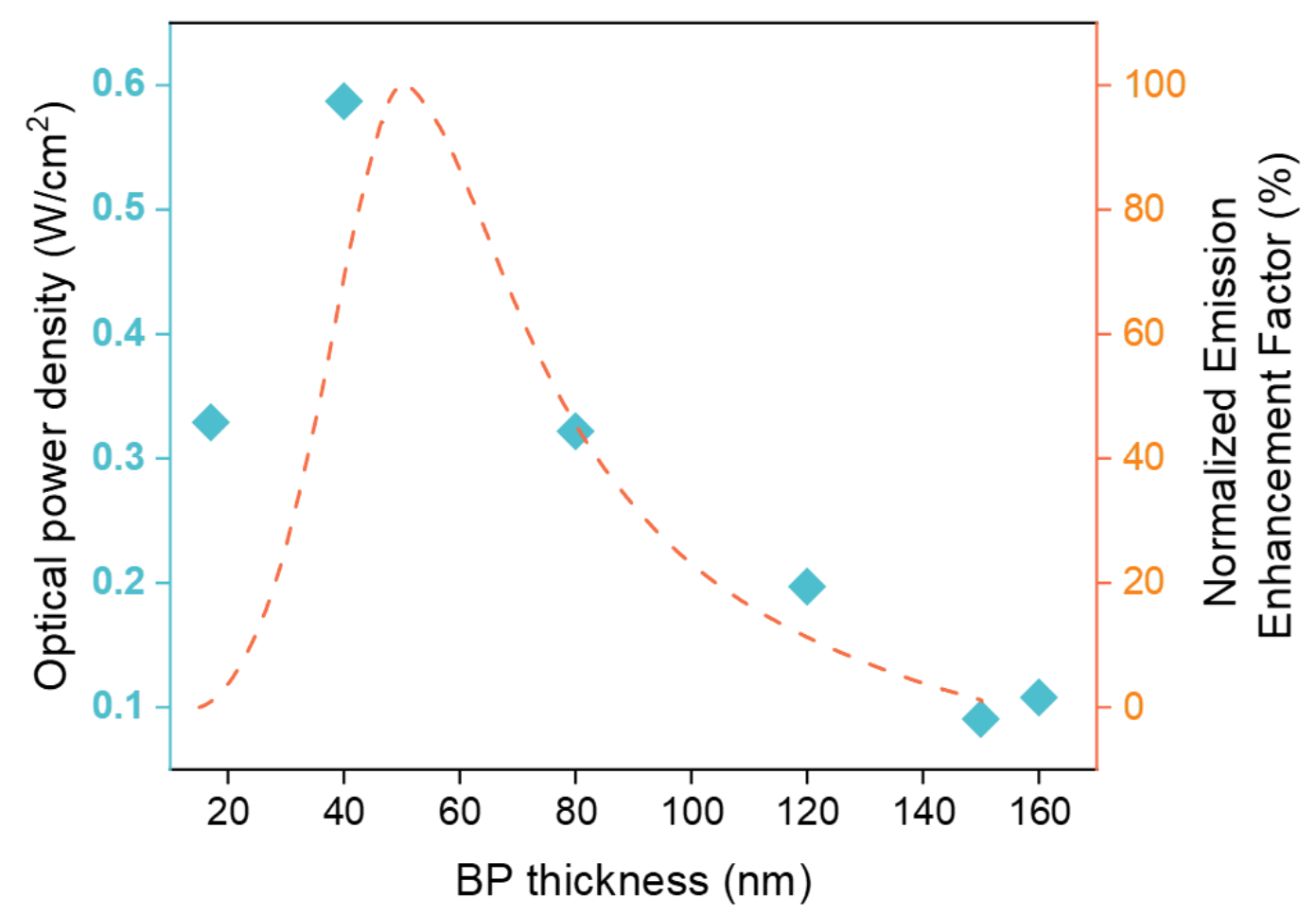

Fig. S4. Effect of bP thickness on the bP-LED output power density for 360nm thick cavity measured at the same current density of $50 \mathrm{Acm}^{-2}$, with the trend overlapped with the normalized emission enhancement factor for the same cavity size. 


\section{S7: ITO Refractive Index Estimation}

The refractive index estimation for ITO was done using the transfer matrix method where for each wavelength, the theoretical reflection was calculation using the equations ${ }^{2}$ :

$$
\left[\begin{array}{l}
B \\
C
\end{array}\right]=\left[\begin{array}{cc}
\cos \delta_{1} & \left(i \sin \delta_{1}\right) / \eta_{1} \\
i \eta_{1} \sin \delta_{1} & \cos \delta_{1}
\end{array}\right]\left[\begin{array}{c}
1 \\
\eta_{2}
\end{array}\right]
$$

where,

$$
\delta_{1}=\frac{2 \pi N_{1} d_{1} \cos v_{1}}{\lambda}
$$

Giving the reflection as,

$$
R=\left(\frac{\eta_{0}-Y}{\eta_{0}+Y}\right)\left(\frac{\eta_{0}-Y}{\eta_{0}+Y}\right)^{*}
$$

Here $Y=C / B$, and $\eta_{0}, \eta_{1}$ and $\eta_{2}$ are the admittances of air, ITO and gold respectively, $v_{1}$ is the refraction angle of light in ITO, and $N_{1}$ is the refractive index of ITO. The admittances and the refraction angles were calculated using Snell's Law, and the model assumed linearly polarized light and an incidence angle to the stack of $20^{\circ}$ (which resulted in negligible difference compared to the case of $0^{\circ}$ incidence angle). The values of the refractive index of evaporated Au were used from the work done by Olmon et al. ${ }^{3}$ The measured reflectance in the infrared range of sputtered ITO with thickness ranging from $50 \mathrm{~nm}$ to $1000 \mathrm{~nm}$ on $\mathrm{Au}$, were carried out using Thermo Fischer Nicolet FTIR Microscope with the IR source and KBr beamsplitter. The refractive index of ITO was then obtained by minimizing the difference between the theoretical and measured reflectance data using least-squares method with the results illustrated in Fig. S5.

2. Macleod, H. A., \& Macleod, H. A. (2010). Thin-film optical filters. CRC press. 
3. Olmon, R. L., Slovick, B., Johnson, T. W., Shelton, D., Oh, S. H., Boreman, G. D., \& Raschke, M. B. (2012). Optical dielectric function of gold. Physical Review B, 86(23), 235147.

a

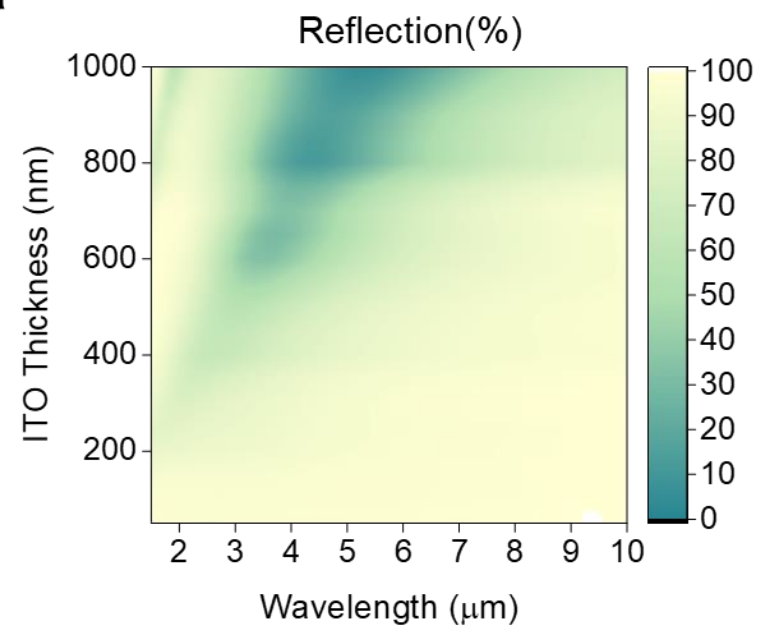

b

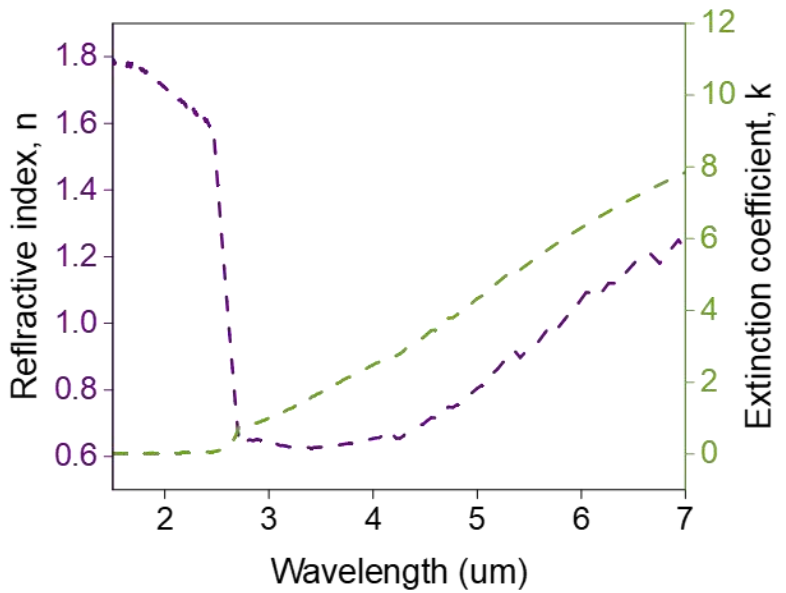

Fig. S5. (a) Measured reflection of ITO with different thickness (50 nm - $1000 \mathrm{~nm})$ on Au in the infrared regime. (b) Fit refractive index of sputtered ITO using least-squares method. 


\section{S8: Series resistance calculation}

a

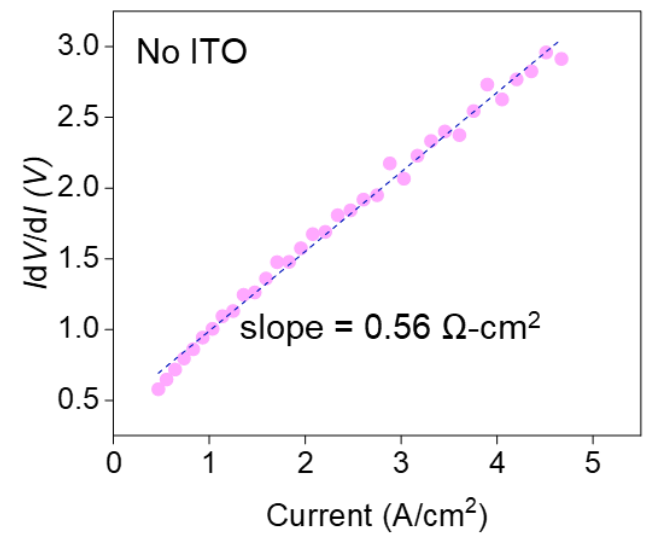

b

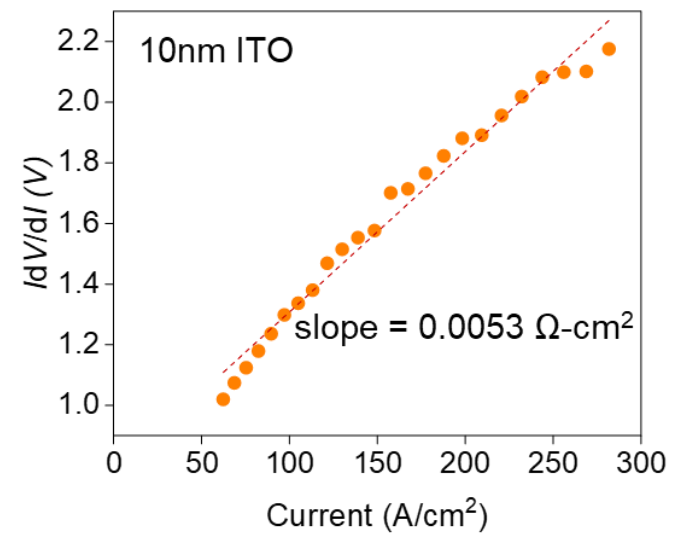

Fig. S6. $I \mathrm{~d} V / \mathrm{d} I$ as a function of $I$ (current normalized by junction area) to find series resistance, slope of the graph for (a) no ITO and, (b) $10 \mathrm{~nm}$ ITO. 


\section{S9: Calibration of Output Power}

The calibration method essentially needed a "counts-to-power" conversion factor which can be used to multiply to the arbitrary counts obtained from the emission spectrum $\left(C_{\mathrm{bP}-\mathrm{LED}}\right.$, area under the spectrum) in the software. For this, we chose a $4.05 \mu \mathrm{m}$ quantum cascade laser (Thorlabs QF4050T1) as the reference light source, reason being the narrow bandwidth and closer emission wavelength to the bP emission wavelength. Since the light from the laser is a directed beam unlike broad distribution beams that most LEDs show, instead of directly putting the laser in front of the objective, the light from the laser was first collimated using a $\mathrm{CaF}_{2}$ lens, then a second $\mathrm{CaF}_{2}$ lens which converged the collimated light, and lastly through a mirror which reflected that converging light and finally reflecting off from a Labsphere reflection standard (setup represented in Fig. S7). The reflection standard reflected the laser light in a Lambertian distribution which afterwards followed the same optical path and electrical instrumentation as bP-LED to collect counts from the laser spectrum $\left(C_{\text {ref }}\right)$. The laser power falling onto the reflector was measured by replacing it with the highly sensitive Ophir Nova $7 Z 01500$ power meter (setup shown in Fig. S8), and this power was divided by the reflectivity of the reflection standard at $4.05 \mu \mathrm{m}$ to find out the power reflecting from it $\left(P_{\text {ref }}\right)$. The counts from the spectrum measurement and the power from the power meter were then used to calculate counts-to-watt conversion factor. To use this factor for finding the bP-LED power, the correction for difference in detector sensitivity for the bP emission wavelength $(3.65 \mu \mathrm{m}) v s$ the laser emission wavelength $(4.05 \mu \mathrm{m})$ was also needed. This called for the evaluation of the instrument function $(I R F)$ of the MCT detector in the FTIR which was evaluated by dividing the measured spectrum to the theoretical spectrum of blackbody at $400^{\circ} \mathrm{C}$. The theoretical spectrum of the black body was calculated using the equation: 


$$
M_{\mathrm{e}}(\lambda, T)=\frac{2 \pi h c^{2}}{\lambda^{5}\left(e^{h c(k \lambda T)^{-1}}-1\right)}
$$

where $M_{\mathrm{e}}$ is Planks law for spectral exitance and $T$ is the temperature. The correction factor was represented as the ratio of the instrument function value at laser wavelength $\left(I R F_{\text {ref }}\right)$ to that at the bP-LED emission wavelength ( $\left.I R F_{\mathrm{bP}-\mathrm{LED}}\right)$. The second correction factor that was required in the calibration process, was for the slight dissimilarity between the angular distribution of the Lambertian reflection standard and bP-LED. The bP-LED angular distribution shown in Fig. S9 was extracted from the far-field monitor in the optical simulations performed using Lumerical FDTD Solutions. This dissimilarity is important because the reflective objective only accepts light at angles ranging from $11.6^{\circ}$ to $35.4^{\circ}$ from the normal incidence. The correction factor was obtained by integrating the light intensity accepted by the objective for laser $\left(A_{\text {ref }}\right)$ and bP-LED ( $\left.A_{\mathrm{bP}-\mathrm{LED}}\right)$, and calculating their ratio. Finally, the device power output, $P$ was found from the following equation:

$$
P=C_{\mathrm{bP}-\mathrm{LED}} \times \frac{P_{\mathrm{ref}}}{C_{\mathrm{ref}}} \times \frac{I R F_{\mathrm{ref}}}{I R F_{\mathrm{bP}-\mathrm{LED}}} \times \frac{A_{\mathrm{ref}}}{A_{\mathrm{bP}-\mathrm{LED}}}
$$

The differences in the FTIR gain and the lock-in-amplifier were also accounted for the power output and efficiency calculations along with the fact that the device was operated at $50 \%$ duty cycle. 


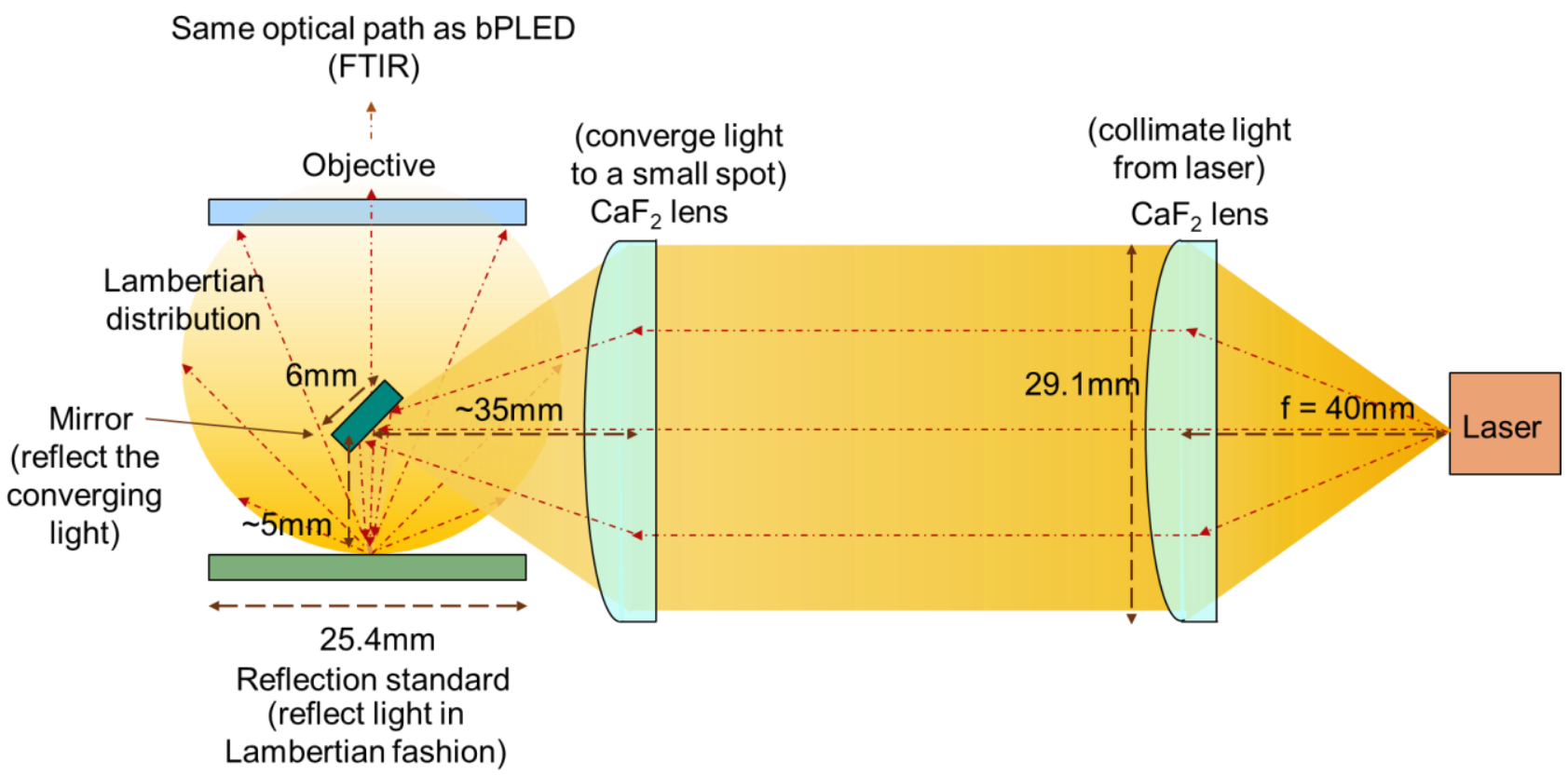

Fig. S7. Schematic of calibration setup to measure the counts of reference laser.

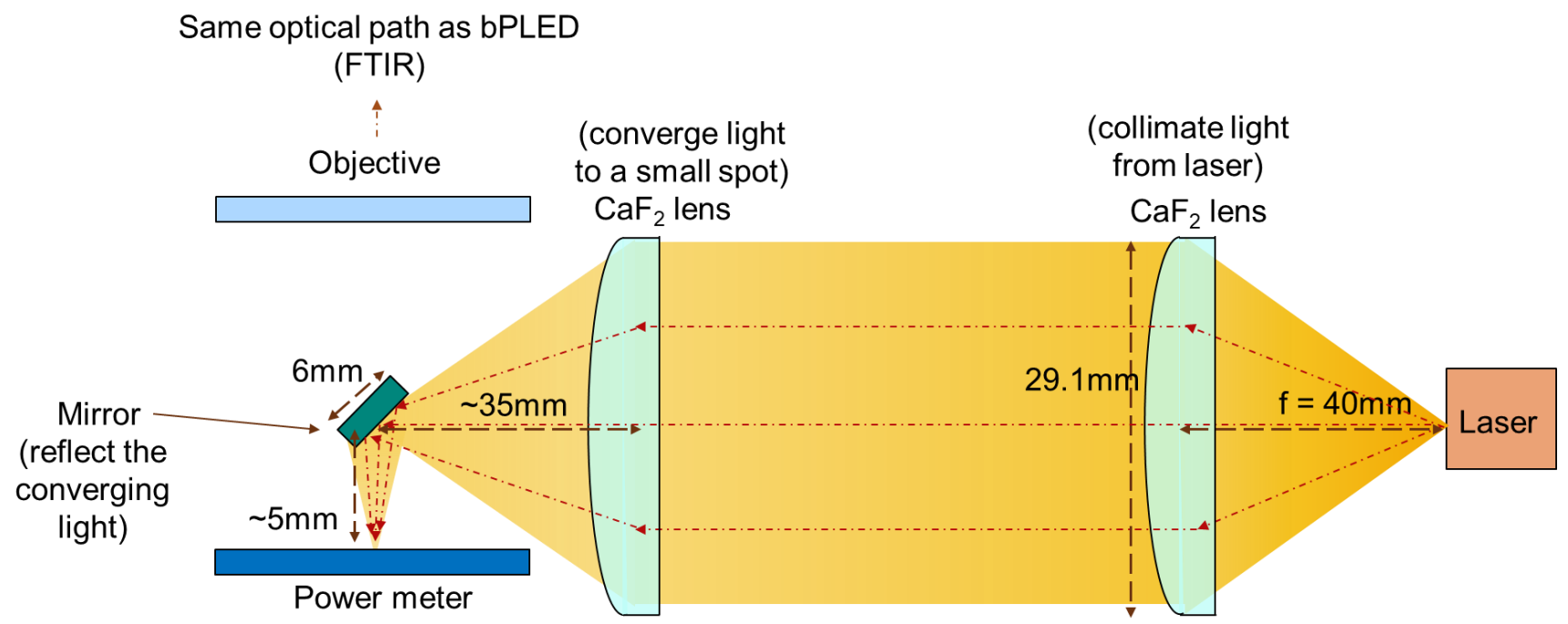

Fig. S8. Schematic of calibration setup to measure power of reference laser. 


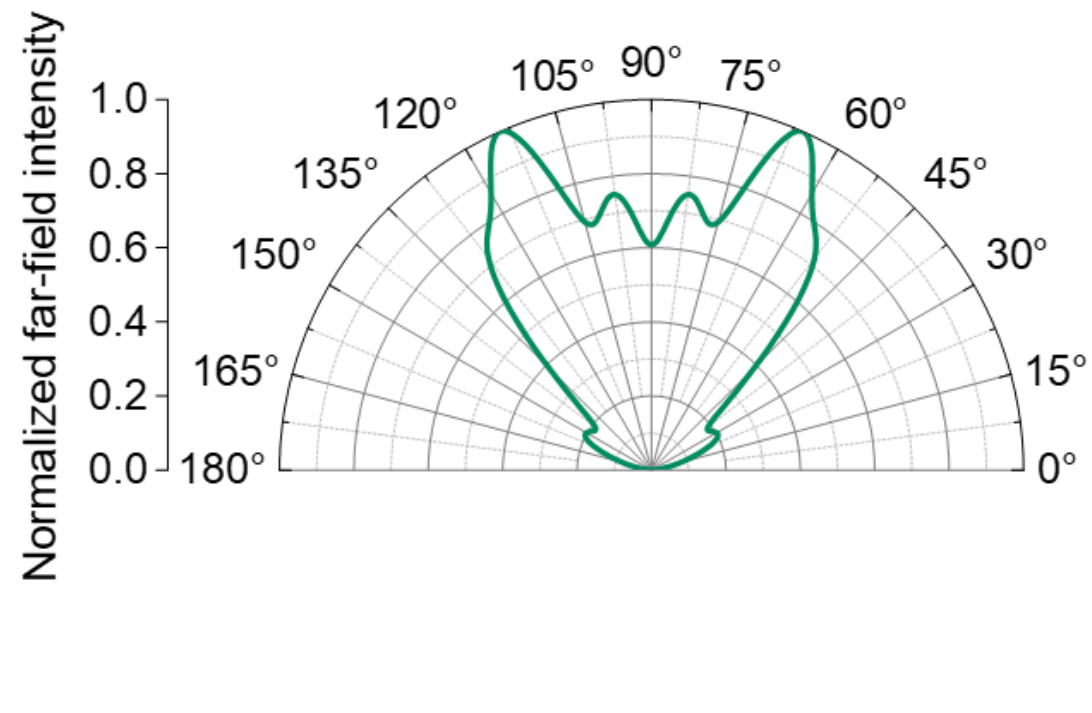

Fig. S9. Angular distribution of bP-LED extracted from optical simulations. 